\title{
Mary Fairfax Greig Somerville
}

\begin{abstract}
Riad HAIDAR haidar@onera.fr

Scientifique, mathématicienne et écrivaine écossaise, Mary Somerville est surtout connue pour avoir traduit et popularisé en Grande-Bretagne la Mécanique Céleste de Laplace. Avec sa contemporaine Caroline Herschel, elles sont les premières à intégrer la prestigieuse Royal Astronomical Society de Londres.
\end{abstract}

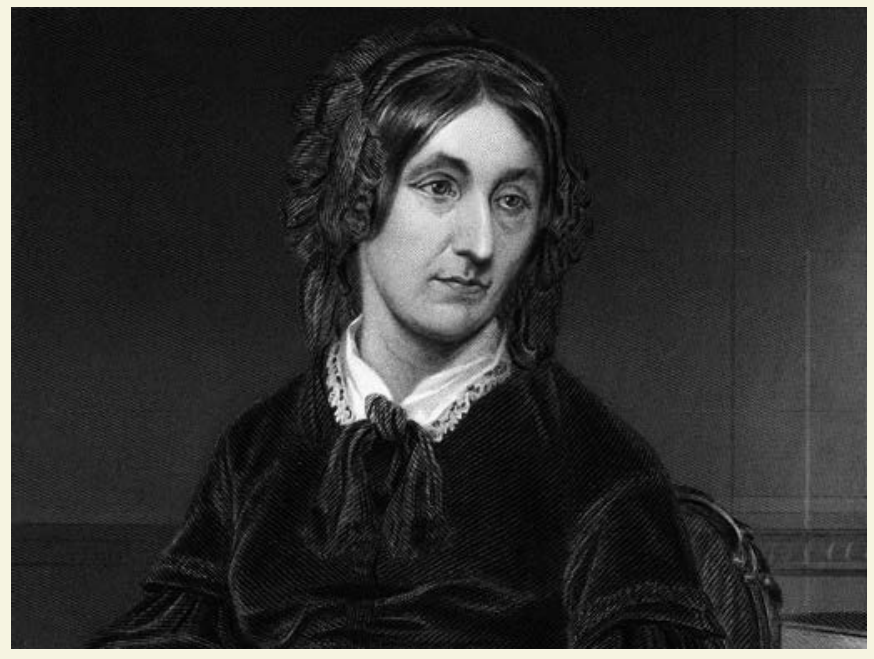

$\mathrm{M}$ ary est la fille de William George Fairfax, officier dans la marine britannique et futur vice-Amiral, et de Margaret Charters. La maison des Fairfax est à Burntisland, une ville du comté de Fife, sur la côte nord du Firth of Forth (littéralement : l'estuaire, ou firth, du fleuve Forth), localisée à l'est d'Edimbourg, en Écosse. Pourtant c'est à Jedburgh que Mary naît le 26 décembre 1780, dans le presbytère de la ville, où loge sa tante Martha Charters qui a épousé le clergyman Thomas Somerville. En effet, Margaret vient de quitter Londres, où son époux s'est embarqué pour un long voyage en mer, et s'est rendue chez sa sœur pour accoucher. Mary est le cinquième de sept enfants, dont seuls quatre survivront. Ses deux frères sont envoyés à l'école mais, comme c'est alors l'usage, Mary et sa sœur sont éduquées à la maison par leur mère, qui leur apprend à lire - pas à écrire... À l'âge de dix ans, Mary est envoyée au pensionnat pour filles de Miss Primrose, qui est établie à Musselburgh, sur la côte sud du Firth of Forth. Elle n'y passe qu'un an, avec le sentiment aigu d'être un animal en cage.

Pourtant, Mary est avide d'apprendre. À son retour à Burntisland, elle se met à lire tout ce qui lui tombe sous la main, malgré la réprobation de certains de ses proches, gênés par cette habitude si peu digne d'une future lady. Mais son oncle, le clergyman Thomas Somerville, lorsqu'il découvre que Mary a appris seule le latin, l'encourage à poursuivre. Elle passera ainsi beaucoup de temps en sa compagnie, dans le presbytère de Jedburgh, en même temps qu'elle se soumet de bonne grâce à l'éducation classique d'une jeune fille:

\section{PRINCIPALES DATES}

\begin{tabular}{|l|l|}
\hline 26 décembre 1780 & Naissance à Jedburgh (Scottish Borders, Écosse) \\
\hline 1831 & Traduction de la Mécanique Céleste de Laplace \\
\hline 1835 & Élection à la Royal Astronomical Society \\
\hline 1848 & Publication de Physical geography \\
\hline 1870 & Victoria Gold Medal de la Royal Geographical Society \\
\hline 29 novembre 1872 & Décès à Naples (Italie)
\end{tabular}

elle apprend la couture et le piano, et se forme à la peinture en compagnie de l'artiste Alexander Nasmyth.

\section{Éveill mathématique}

C'est précisément Nasmyth qui éveille, par hasard, son intérêt pour les mathématiques, un jour qu'il évoque les Éléments d'Euclide pour introduire la notion de perspective en peinture, et dont il dit qu'ils permettent la compréhension de l'astronomie et d'autres sciences. Piquée de curiosité, Mary décide dès lors de s'initier à l'œuvre du maître antique avec l'aide du tuteur de son jeune frère, qui lui inculque également des rudiments d'algèbre. Les mathématiques deviennent très vite une passion si dévorante que Mary y consacre tout son temps libre, la nuit comme le jour, sans compter les heures - à tel point que ses parents finissent par s'inquiéter pour sa santé. Mais Mary persiste, et poursuit ses veillées studieuses en secret.

En 1804, elle épouse le capitaine Samuel Greig, son cousin éloigné du côté de sa mère, officier dans la marine russe et qu'elle fréquente depuis quelque temps. Il vient d'être nommé consul de Russie en Angleterre, et le couple s'installe à Londres. Mais Samuel décède brusquement trois ans après leur mariage et Mary retourne en Écosse, avec leurs deux enfants, dont Woronzow Greig, qui deviendra avocat et scientifique. Elle y retrouve un cercle d'amis qui l'encouragent à poursuivre ses études en sciences - ce qu'elle fait, l'héritage de Samuel la mettant à l'abri du besoin. Parmi ses 
relations, John Playfair, professeur de philosophie naturelle à Edimbourg, la met en contact avecl'un de ses anciens élèves, William Wallace, lui-même professeur de mathématiques au Collège militaire royal de Great Marlow, avec lequel elle découvre les problèmes posés dans le Mathematical Repository, un almanach publié à la fin du $18^{\mathrm{e}}$ siècle en Angleterre. Sur les conseils de Wallace, Mary lit les Principia de Newton ainsi que la Mécanique Céleste de Laplace...

En 1812, Mary épouse son autre cousin William Somerville, le fils de sa tante Martha et du clergyman Thomas, avec qui elle aura quatre enfants. Médecin et inspecteur des hôpitaux, William est un esprit cultivé et ouvert, et il encourage sa femme à poursuivre ses travaux mathématiques. Ils s'installent à Edimbourg, au sein d'un groupe d'amis et intellectuels, dont le physicien David Brewster. En 1814, Mary perd coup sur coup sa fille aînée, née de son premier mariage, et le fils qu'elle vient d'avoir...

En 1816 William, promu inspecteur au Conseil médical de l'armée, est élu à la Royal Society et la famille déménage d'Edimbourg à Londres. Les époux Somerville intègrent alors l'un des cercles intellectuels les plus brillants et influents de l'époque, et fréquentent Airy, John et William Herschel, ainsi que de nombreux scientifiques européens en visite à Londres. En 1817 ils se rendent à Paris, où ils sont reçus par Biot, Arago, Laplace et Poisson. En 1824, William est affecté comme médecin à l'Hôpital royal de Chelsea; les Somerville s'établissent alors dans la banlieue londonienne.

\section{Travaux scientifiques}

C'est à cette époque que Mary entreprend ses premières investigations scientifiques : elle mène en 1825 des expériences originales sur le magnétisme, dont elle synthétise les résultats dans une note qu'elle présente en 1826 à la Royal Society. Cette note est historique car, avec les travaux de Caroline Herschel, elle fait partie des tout premiers papiers écrits par une femme à être publiés dans les Philosophical Transactions.

En 1827 Lord Henry Brougham demande à Mary Somerville, au nom de la Society for the Diffusion of Useful Knowledge, de traduire la Mécanique Céleste de Laplace. Il s'agit d'un travail considérable, qui demande un effort particulier car les notions mathématiques utilisées par Laplace sont alors inconnues de la plupart des mathématiciens anglais. Mary accepte la mission, et s'en acquitte au-delà des espoirs de ses commanditaires : bien plus qu'une simple traduction, elle réussit le tour de force de rendre accessible l'œuvre du mathématicien français. Mais le texte traduit, qu'elle a enrichi de nombreuses digressions pédagogiques, est beaucoup trop volumineux pour être publié par la Society qui l'a commandité et John Herschel le recommande à l'éditeur John Murray. Le livre, qui paraît en 1831, est un succès immédiat et considérable, et Mary acquiert une grande notoriété dans les milieux scientifiques. Elle passe ensuite un an sur le continent, entre 1832 et 1833 , en majeure partie à Paris. Elle y travaille sur son prochain livre The connection of the physical sciences, qui est publié en 1834 . Ses échanges avec les mathématiciens français sont nombreux et fructueux : il est par exemple remarquable qu'en 1836, Arago fait publier un extrait d'une lettre de Mary dans les Comptes Rendus de l'Académie des Sciences.

Mary reçoit désormais de nombreuses marques de reconnaissance. Elle devient membre honoraire de la Société de Physique et d'Histoire Naturelle de Genève en 1834 et, la même année, à la Royal Irish Academy. Elle est élue à la Royal Astronomical Society en 1835, et Robert Peel, alors Premier ministre britannique, lui accorde une pension civile de $200 £$ par an. Cette pension, déjà confortable, est augmentée à $300 £$ en 1837 par son successeur, William Lamb.

\section{Ultima verbae}

En 1838, la santé de William se dégrade brusquement et la famille s'installe définitivement en Italie, dont le climat lui est plus favorable; et en effet, William survivra encore 22 années. Mary écrit de nombreux ouvrages, dont certains influenceront fortement James Maxwell. Son texte le plus abouti, et son plus grand succès, est Physical geography qui paraît en 1848 et qui sera enseigné en université jusqu'au début du $20^{\circ}$ siècle. Il vaut à Mary de nombreux honneurs, dont l'élection à l'American Geographical and Statistical Society en 1857 et, en 1870, la Victoria Gold Medal de la Royal Geographical Society.

Personnalité engagée, elle met sa notoriété au service de la cause des femmes et signe en 1868 la pétition de John Stuart Mill, philosophe et économiste britannique, pour le suffrage des femmes. En 1869 elle achève son autobiographie, dont sa fille Martha publiera des extraits après sa mort. Atteinte de surdité et rendue frêle par le grand âge, Mary n'en conservera pas moins une grande vivacité d'esprit jusqu'à sa mort paisible, à l'âge de 92 ans. Elle est enterrée au cimetière anglais de Naples.

\section{POUR EN SAVOIR PLUS}

A. Chapman, Mary Somerville and the World of Science (Canopus Publishing Limited, 2004).

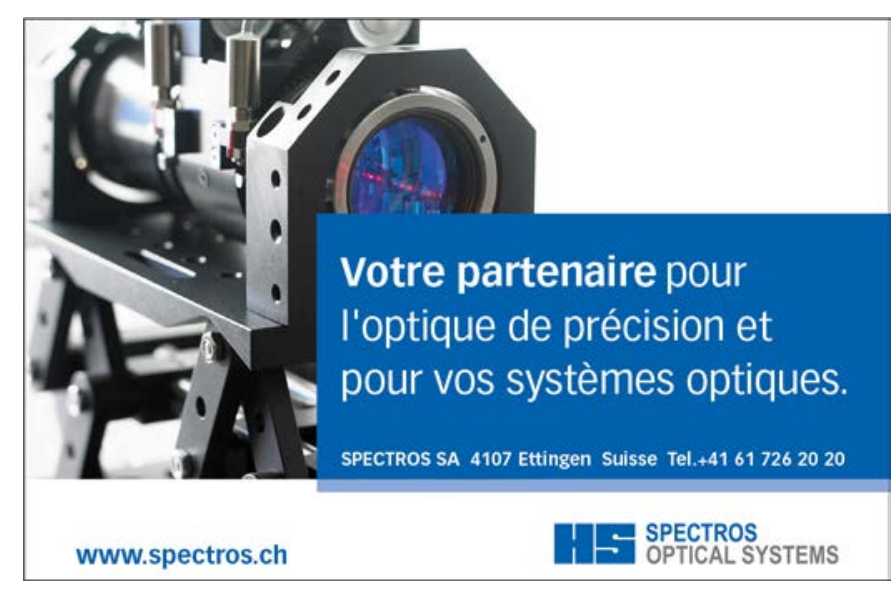

